\title{
À la rencontre des voix francophones dans la ville de Québec : les attitudes des Québécois à l'égard de diverses variétés de français
}

\author{
Adéla Šebková ${ }^{1,}$, Kristin Reinke $^{2}$, et Suzie Beaulieu ${ }^{3}$ \\ ${ }^{1}$ FLSH, Université Laval, Québec, Canada \& École doctorale IV, Sorbonne Université, Paris, France \\ ${ }^{2}$ FLSH, Département de langues, linguistique et traduction, Université Laval, Québec, Canada \\ ${ }^{3}$ FLSH, Département de langues, linguistique et traduction, Université Laval, Québec, Canada
}

\begin{abstract}
Résumé. Le Québec mise actuellement sur un accroissement des communautés immigrantes francophones pour faire face au vieillissement de la population et pour combler une rareté de main-d'œuvre. Ainsi, des villes traditionnellement homogènes, comme la ville de Québec, voient leur paysage ethnoculturel se diversifier considérablement et rapidement, ce qui donne lieu à des débats sur la diversité culturelle grandissante et la place de l'immigration. Dans ce contexte, il importe de documenter les attitudes, positives ou négatives, de ses habitants à l'égard des variétés de français parlées par les nouveaux arrivants, marquées par un accent francophone étranger. Pour ce faire, nous utilisons une méthodologie inspirée du test du locuteur masqué, combinée avec un questionnaire, afin de faire évaluer la personnalité, les compétences, la compréhensibilité, la correction de la prononciation des locuteurs francophones ainsi que la volonté de nos participants d'établir des liens avec ces derniers dans un contexte amical, professionnel et public. Les résultats montrent que les attitudes sont généralement positives, mais qu'elles changent en fonction de la variété, et que les voix les mieux évaluées sont celles des Québécois.
\end{abstract}

\begin{abstract}
Meeting the French-speaking voices in Quebec City: Quebecers' attitudes towards various varieties of French. Quebec is currently relying on an increase of French-speaking immigrant communities to cope with an ageing population and to fill labor shortages. Thus, the ethnocultural landscape of traditionally homogeneous cities is diversifying considerably and rapidly, giving rise to debates about the growing cultural diversity and the place of immigration. In this context, it is important to document the attitudes, positive or negative, of its citizens towards the varieties of French spoken by newcomers, marked by a foreign French accent. For this purpose, we use a methodology inspired by the matched-guise test, combined with a questionnaire, to assess the personality, skills, comprehension, correctness of the pronunciation of French-speaking voices and the willingness of our participants to connect with them in a private, professional and public context. The results have
\end{abstract}

*Corresponding author: adela.sebkova.1@ulaval.ca 
shown that attitudes are generally positive but differ depending on the French variety; and that the best-rated voices are those of Quebecers themselves.

\section{Problématique et objectifs}

Le Canada est une société multiculturelle qui a été façonnée au fil du temps par les immigrants et leurs descendants. Quant au Québec, la législation fédérale l'autorise à choisir de façon autonome la moitié de ses immigrants. Étant la seule province dont l'unique langue officielle est le français, il n'est pas surprenant que le Québec favorise les immigrants francophones afin de protéger le caractère francophone du Québec (Immigration, Réfugiés et Citoyenneté Canada, 2019). Si, dans le passé, le Québec et notamment la ville de Québec étaient plutôt un lieu de passage transitoire pour les immigrants, la situation économique favorable actuelle les encourage à s'y installer définitivement (Bourque, 2018). Étant donné la forte progression du flux migratoire, le Québec se préoccupe davantage des mesures qui favorisent l'intégration des nouveaux arrivants dans la société québécoise (Bourque, 2018).

Il est bien établi que le processus d'intégration des immigrants dépend, entre autres, des relations intergroupes, notamment des attitudes des membres de la société d'accueil à l'égard des immigrants (Ochs et Schieffelin, 2017). À cet égard, "l'effet de la langue sur le jugement social ${ }^{i}$ (Giles et Billings, $2004: 187$ ) n'est plus à démontrer. En effet, les recherches sur les attitudes langagières montrent que les locuteurs portent un jugement sur leurs interlocuteurs, notamment, sur la base de leur façon de parler (Dragojevic et al., 2013; Lambert et al., 1960). Afin d'accéder aux attitudes langagières d'une population, la technique du locuteur masqué a été développée par des chercheurs canadiens en psychologie sociale; elle est devenue l'outil de mesure par excellence des attitudes langagières (Lambert et al., 1960). Dans sa mouture originale, cette technique consiste à présenter des enregistrements (stimuli) réalisés par les mêmes locuteurs utilisant successivement des langues différentes. Les participants évaluent, à partir de la voix, des attributs personnels en rapport avec les facteurs solidarité intergroupe (p. ex. sympathie) et statut socioéconomique (p. ex. compétence professionnelle). Comme les participants ne sont pas conscients qu'ils évaluent chaque fois le même locuteur, leurs évaluations sont censées refléter indirectement leurs attitudes à l'égard des langues évaluées et, croit-on, des groupes qui les parlent.

Si de nombreuses recherches utilisant cette technique ont porté sur le rapport entre le français et l'anglais (Lambert et al., 1960; Genesee et Holobow, 1989; Laur, 2008; Rousseau, 2012; Kircher, 2014), la méthode, telle quelle ou adaptée, a aussi été utilisée pour mieux comprendre le rapport entre les variétés de français, et le Québec est un milieu propice pour observer ces dynamiques. En effet, rappelons que le Québec est depuis longtemps un endroit caractérisé par la concurrence entre deux normes de référence : d'une part, la norme de référence locale, endogène - la norme de la variété québécoise standard considérée par les Québécois eux-mêmes comme neutre dans des situations de communication formelles telles que l'information -, et d'autre part, la norme de référence traditionnelle - la norme exogène, française, qui correspond au modèle langagier du français des Parisiens cultivés. En fait, les débats sur la norme du français et la qualité de la langue au Québec sont très anciens, mais les deux normes, québécoise et française, sont encore de nos jours en concurrence. Cette situation a généré pendant longtemps un sentiment d'insécurité linguistique ${ }^{\mathrm{ii}}$ chez les Québécois ${ }^{\mathrm{iii}}$ et a influencé leurs attitudes envers leurs propres façons de parler (Remysen, 2018). 
En effet, les premières études ayant utilisé la technique du locuteur masqué, parfois combinées avec d'autres méthodes de collecte de données (p. ex. questionnaire, entrevue semi-dirigée), ont mis en évidence l'existence d'un malaise chez les Québécois par rapport à leurs variétés de langue, notamment familière (Preston, 1963; Laberge et ChiassonLavoie, 1971; d'Anglejan et Tucker, 1973; Méar-Crine et Leclerc, 1976; Noël, 1980; Lappin, 1982). Les études ont également montré que les locuteurs utilisant des variétés standard tendent à être associés aux groupes dominants du point de vue socioéconomique et, par conséquent, tendent à être évalués plus favorablement quant au facteur statut (Fuertes et al., 2012), ce qui traduirait le prestige manifeste d'une variété. En revanche, les variétés non standard reçoivent des appréciations plus favorables quant à la solidarité (Ryan, 1979; Lafontaine, 1997), ce qui traduirait le prestige latent d'une variété.

À partir des années 1980, les recherches sur les attitudes ont adopté d'autres voies méthodologiques, ciblant les attitudes par rapport à des variantes linguistiques spécifiques en fonction de leur valeur sociale. Ainsi, les études de Lappin (1982), Tremblay (1990) et Reinke (2000) ont démontré que certaines variantes phonétiques typiques du français québécois (désormais FQ), comme l'affrication de /t/ et /d/ devant/i/ et /y/ (p. ex. pet $t_{s} i$ ), ne suscitaient pas de jugements défavorables, tandis que d'autres étaient toujours critiquées, notamment celles associées aux locuteurs de milieux défavorisés, comme la prononciation [we] en fin de mot (p. ex. moé). Il semble donc y avoir un consensus autour de certaines variantes socialement valorisées au Québec, ce qui suggère l'émergence d'une norme proprement québécoise prônant une variété québécoise de prestige. Les travaux plus récents de Laur (2001), Evans (2002), Remysen (2004) et Chalier (2014 et 2018) confirment que les attitudes des Québécois à l'égard de leur français sont plus positives qu'elles ne l'étaient. De plus, dans une étude récente cherchant à savoir si les Québécois favorisent une norme standard "parisienne » ou une norme québécoise, Chalier (2018) démontre que la prononciation est le facteur le plus important dans l'évaluation des stimuli. Si, jusqu'à tout récemment, les sociolinguistes décrivaient la valeur sociale positive de certaines variantes québécoises par le terme labovien de prestige latent (Reinke 2000; Pöll, 2005 et 2017), Chalier (2018) et Pustka et al. (2019) soutiennent que des prononciations québécoises faiblement marquées bénéficient aujourd'hui d'un prestige manifeste.

Dans une perspective panfrancophone, certaines études se sont intéressées aux perceptions des accents francophones (Moreau et al., 2007; Goldman et al., 2014; Lazaridis et al., 2014; Avanzi, 2016; Avanzi et Boula de Mareüil, 2017 et 2019; Pustka et al., 2019). Les résultats confirment l'existence d'une pluralité normative au sein de la francophonie. A titre d'exemple, l'étude de Moreau et al. (2007), réalisée dans 6 pays francophones (France, Québec, Belgique, Suisse, Sénégal et Tunisie), démontre que la norme hexagonale n'occupait plus une position privilégiée dans l'imaginaire collectif et que, en revanche, la norme préférée par les auditeurs était la norme nationale (norme endogène) associée aux groupes des locuteurs scolarisés.

Pour revenir aux attitudes langagières des Québécois dont l'étude se trouve au cœur de notre recherche, on constate que très peu d'études se sont intéressées jusqu'à présent à la perception des autres variétés diatopiques du français avec lesquelles la population québécoise était en contact, mise à part la variété hexagonale. De plus, les chercheurs ont porté une attention particulière à Montréal, à quelques exceptions près, ce qui n'est pas surprenant, la métropole étant plus hétérogène du point de vue linguistique et ethnique que le reste du Québec (Remysen et Reinke 2014 : iii). Par conséquent, il nous semble judicieux d'étudier les attitudes de la population de la ville de Québec, d'autant plus que la capitale nationale connait une grande augmentation de l'immigration. En effet, dans un contexte de diversité ethnoculturelle grandissante, Québec est désormais un lieu où se croisent plusieurs variétés diatopiques de français. Il importe donc de documenter le capital linguistique conféré aux différents français dans ce marché linguistique, capital qui se concrétise dans 
les réactions mesurables des locuteurs sur leurs propres pratiques langagières et sur celles des autres.

L'objectif de notre contribution est d'identifier les attitudes des participants québécois à l'égard des variétés de français parlées par les quatre communautés francophones les plus représentées dans la ville de Québec (française, haïtienne, algérienne et camerounaise) et à l'égard de leur propre variété. Plus spécifiquement, il s'agit de savoir si certaines de ces variétés reçoivent des évaluations plus positives que d'autres. Autrement dit, notre objectif est de révéler une éventuelle hiérarchisation normative à l'égard de ces variétés. Adoptant une démarche empruntée aux études sociolinguistiques antérieures menées dans le contexte canadien (Lambert et al., 1960; d'Anglejan et Tucker, 1973, Genesee et Holobow, 1989, etc.) notre étude s'inscrit, d'une part, dans la tradition des études explorant les attitudes des Québécois à l'égard du français hexagonal et du FQ, et d'autre part, elle prolongera la réflexion en incluant d'autres variétés diatopiques de français, ce qui, à notre connaissance, n'a pas encore été exploré dans le contexte québécois.

\section{Méthodologie}

\subsection{Le test d'attitudes}

Afin d'accéder aux attitudes langagières de nos participants, nous avons construit un test d'attitudes divisé en trois parties, débutant par un test composé de dix extraits audios destinés à l'écoute des participants, suivi d'un questionnaire à réponse unique et complété par un questionnaire sociodémographique.

Pour le test composé d'extraits audios (partie 1), nous avons utilisé une méthodologie appelée verbal guise, inspirée par des travaux de Lambert et al. (1960). En effet, il aurait été impossible d'adopter la technique du locuteur masqué telle que nous venons de la définir dans l'introduction, étant donné qu'il est irréaliste de prétendre que des locuteurs francophones soient capables d'imiter à la perfection la prononciation des cinq variétés à l'étude. C'est la raison pour laquelle nous avons opté pour une méthode qui fait appel à différents locuteurs ayant tous des caractéristiques semblables, représentatifs de chacune des variétés étudiées. Une telle méthode indirecte permet d'explorer davantage l'inconscient des gens (McKenzie, $2010: 86$ ), puisque c'est là que les attitudes langagières opèrent le plus souvent (Harrison, 2014 : 259 ; Lindemann et al., 2014 ; Kang et Rubin, 2014). Pour le reste, notre test ressemble à celui du test du locuteur masqué : nous avons demandé aux juges d'évaluer sur une échelle de Likert à 6 degrés (de " peu » à « très ») les caractéristiques présumées de chacune des personnes enregistrées selon les facteurs solidarité et statut. Pour chaque facteur, nous avons choisi les trois attributs les plus fréquemment utilisés dans les études antérieures afin d'assurer la comparabilité de nos résultats, à savoir la sympathie, le dynamisme et la sociabilité pour la solidarité, ainsi que le professionnalisme, le degré d'instruction et l'aptitude à diriger pour le statut. Nous avons choisi une échelle à 6 degrés pour nous assurer que les juges prennent parti dans leurs évaluations et qu'ils ne choisissent pas la valeur médiane, comme cela aurait pu être le cas si nous avions choisi une échelle à 5 degrés.

En ce qui concerne le questionnaire à réponse unique (partie 2), d'autres éléments associés à des jugements de valeur sur les pratiques langagières des individus ont été explorés. Premièrement, nous avons demandé aux juges d'évaluer, sur la même échelle, la compréhensibilité des stimuli et leur perception de la correction de la prononciation des personnes écoutées dans la première partie du test En effet, il a été démontré que certaines prononciations sont jugées comme étant incompréhensibles en raison de leur ancrage « local» ou «folklorique » (Moreau, $2007: 35$; Ostiguy et Reinke, $2015: 242$ ), ou bien 
comme étant linguistiquement incorrectes (Paveau, 2008), c'est-à-dire déviant de la norme comprise par les locuteurs d'autres variétés (Moreau, 2007 : 31). Par la suite, les juges ont été invités à exprimer leur accord ou désaccord quant à la volonté d'établir des liens amicaux et professionnels avec les personnes entendues et quant à la possibilité que la personne entendue puisse être enseignant de français. Ces trois situations de communication sont associées à un degré de formalité différent et à un prestige qui leur est propre (Labov, 1976). L'objectif est donc de savoir quelle variété serait, selon nos juges, la plus conforme à telle ou telle situation.

Quant au questionnaire sociodémographique (partie 3), celui-ci avait pour objectif de recueillir les données des participants permettant leur identification. Il s'agit des données telles que le sexe, l'âge, le niveau de scolarité et le lieu de résidence. Il est important de souligner que les juges ne connaissaient pas l'origine géographique des locuteurs, car nous voulions éviter l'influence de leurs croyances sociopolitiques vis-à-vis de l'ethnicité des locuteurs. En effet, il a été démontré à plusieurs reprises que l'origine des locuteurs avait une influence sur les attitudes des juges (Yook et Lindemann, 2013 ; Lindemann, Litzenberg et Subtirelu, 2014 ; Kang et Rubin, 2014).

Dans le souci de ne pas introduire un biais dès le début de l'expérience, nous avons présenté notre étude aux répondants comme une expérience qui avait pour objectif d'observer comment on arrive à évaluer la personnalité et les compétences des personnes enregistrées seulement en écoutant leur voix.

\subsection{Choix et présentation des stimuli}

Afin de constituer notre corpus de stimuli, nous avons demandé à deux Québécois originaires de la ville de Québec et à huit locuteurs franco-dominants non québécois originaires des pays les plus représentés à Québec selon le dernier recensement de Statistique Canada (France, Haïti, Cameroun et Algérie ${ }^{\text {iv }}$ ) de lire un court texte et nous les avons enregistrés avec leur consentement. Tous les locuteurs (cinq femmes et cinq hommes) étaient âgés de 25 à 30 ans et détenaient un diplôme universitaire. Nous avons choisi cette catégorie d'âge parce que, selon le portrait statistique, les immigrants appartiennent majoritairement à ce groupe, disponible à l'emploi (Bourque, 2018).

Inspirées de Lindemann (2003), nous avons demandé aux 10 participants de lire, de la manière la plus naturelle possible, le message d'accueil entendu sur le répondeur téléphonique d'un restaurant. La lecture naturelle est utilisée dans la plupart des études récentes utilisant cette méthode (p. ex. Nejjari et al., 2019), puisqu'elle permet de s'assurer que les différences dans l'évaluation des locuteurs sont liées seulement à leur prononciation et non au contenu du message (Avanzi et Boula de Mareüil, 2017 : 23). Les stimuli étaient d'une durée moyenne de 30 secondes et ont été présentés aux juges de manière aléatoire. Une seule écoute de chaque enregistrement était permise de manière à obtenir les réactions les plus spontanées possible, pour ainsi refléter les jugements de valeur, positifs ou négatifs, associés aux différentes voix entendues.

\section{3 Échantillon de répondants et collecte de données}

Les répondants de notre étude, ci-après dénommés juges, sont des résidents de la ville de Québec âgés de 20 à 30 ans, tous étudiants à l'Université Laval, au nombre total de 24 (14 femmes et 10 hommes). Il s'agit donc d'un échantillon homogène du point de vue sociodémographique (âge, scolarité, provenance géographique), ce qui constitue une des limites de notre étude dont les résultats ne peuvent pas être généralisés à l'ensemble de la population québécoise. Les juges, recrutés via le réseau universitaire et sur les réseaux sociaux, ont répondu à notre test en ligne, conçu à l'aide des formulaires Google. 


\section{Analyse et interprétation des résultats}

\subsection{Test d'attitudes : évaluation des attributs personnels en fonction de la variété}

Pour savoir si les attitudes à l'égard des voix enregistrées étaient positives ou négatives, nous avons calculé, pour chaque attribut, la moyenne des cotes attribuées à chaque groupe de francophones (hommes et femmes confondus ${ }^{\mathrm{v}}$ ) sur un total de 24 juges. Nous considérons que les moyennes obtenues sur une échelle de 1 à 6 correspondent à la distribution suivante : attitudes négatives $<3,50$; attitudes positives $>3,51$. Le tableau 1 présente les moyennes obtenues dans l'ordre décroissant et démontre que les attitudes à l'égard des voix francophones sont généralement positives, c'est-à-dire que, dans la majorité des cas, les moyennes sont supérieures à 3,50, tant par rapport à la solidarité que par rapport au statut.

\begin{tabular}{|l|c|c|c|c|c|}
\hline Solidarité & Québec & Haïti & France & Cameroun & Algérie \\
\hline Sympathique & 4,98 & 4,44 & 3,79 & 4,23 & 3,54 \\
\hline Dynamique & 4,71 & 4,06 & 3,15 & 3,29 & 3,19 \\
\hline Sociable & 4,94 & 4,38 & 3,52 & 3,98 & 3,40 \\
\hline \hline Moyenne (N=48) & 4,88 & 4,29 & 3,49 & 3,83 & 3,38 \\
\hline Statut & & & & & \\
\hline \hline Professionnel & 5,19 & 4,29 & 4,75 & 3,73 & 3,88 \\
\hline Instruit & 4,71 & 4,27 & 4,44 & 3,81 & 4,08 \\
\hline Apte à diriger & 4,92 & 4,04 & 3,96 & 3,40 & 3,56 \\
\hline \hline Moyenne (N=48) & 4,93 & 4,20 & 4,38 & 3,65 & 3,85 \\
\hline \hline & & & & & \\
\hline Moyennes globales & $\mathbf{4 , 9 1}$ & $\mathbf{4 , 2 5}$ & $\mathbf{3 , 9 4}$ & $\mathbf{3 , 7 4}$ & $\mathbf{3 , 6 1}$ \\
\hline
\end{tabular}

Tableau 1. Moyennes globales par groupe.

Afin de savoir si la différence entre les moyennes obtenues était significative, nous avons effectué des tests $t$ pour échantillons indépendants avec le logiciel SPSS, en fixant la valeur $p$ à $0,05^{\text {vi }}$. Les résultats détaillés des analyses statistiques figurent en annexe (voir tableaux 1a, 1b, 1c, 1d). Ils révèlent que les voix québécoises obtiennent des moyennes significativement plus élevées que tous les autres groupes dans la plupart des catégories, à l'exception de l'attribut instruit (relié au facteur statut) pour lequel la différence avec les voix françaises n'est pas significative (valeur $p=0,153$ ). Cette observation peut sans doute être interprétée comme une trace de l'ancien prestige de la norme de référence française/parisienne. Rappelons que, si les premières études sur les attitudes des Québécois par rapport à leur français ont démontré une dévalorisation quant aux deux facteurs statut et solidarité (Lambert et al., 1960 ; d'Anglejan et Tucker, 1973), les travaux qui ont suivi ont graduellement fait apparaître une meilleure évaluation quant à la solidarité (Genesee et Holobow, 1989), signe d'un prestige latent du FQ. Seuls les tout derniers travaux (Chalier, 2018 ; Pustka et al., 2019) dévoilent un prestige manifeste des prononciations québécoises faiblement marquées. Dans le cas de nos juges, on observe une nette amélioration des attitudes au niveau du statut (relié au prestige manifeste), mais avec une certaine réserve concernant le degré d'instruction projeté par la variété parlée.

En ce qui concerne les autres voix testées, les résultats sont moins univoques. Autrement dit, il n'y a pas de différence significative, de manière générale, entre celles 
classées aux troisième, quatrième et dernière position. C'est la raison pour laquelle aucune hiérarchie systématique ne peut être formulée à cet égard.

Malgré l'absence d'une hiérarchisation nette, les résultats du test $\mathrm{t}$ permettent de dégager d'autres tendances quant aux facteurs solidarité et statut ${ }^{v i i}$, éclairant ainsi davantage la prise de position des juges. En effet, on note que le facteur statut obtient des cotes significativement plus élevées que les traits faisant référence à la solidarité (valeur $\mathrm{p}$ $\left.=0,03^{*}\right)$. Nous pouvons ainsi en déduire que les juges sont plus sévères et plus prudents dans leur attribution des notes liées à l'identité de la personne qu'ils ne le sont dans leur attribution des notes liées à ses compétences. À cet égard, une autre observation mérite d'être commentée: le tableau 1 met en évidence que les voix françaises obtiennent généralement de meilleures cotes que les voix haïtiennes, camerounaises et algériennes pour ce qui est du statut, mais elles sont, dans la majorité des cas, moins bien notées en ce qui concerne la solidarité. La figure 1 ci-dessous permet de faire ressortir de façon plus claire cet écart flagrant.

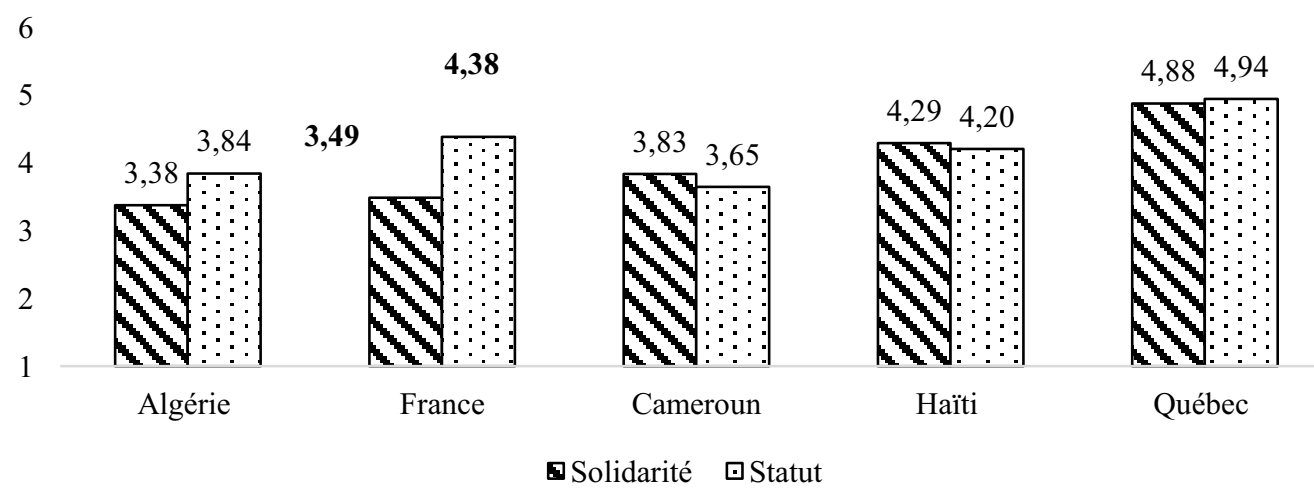

Fig. 1. Moyennes globales obtenues par groupe.

Par ailleurs, la figure 1 démontre que les moyennes des deux facteurs sont relativement proches pour tous les groupes, à l'exception des voix françaises. Cette particularité rejoint les résultats d'une récente étude de Pustka et al. (2019) sur les perceptions des accents au Québec : l'accent parisien a été jugé, d'un côté, le moins sympathique (facteur solidarité), et de l'autre, le plus sérieux (facteur statut). Si le poids normatif traditionnel de la France peut expliquer l'évaluation positive quant au facteur statut, les réactions des juges relatives au facteur solidarité mettent en évidence toute l'importance de la langue en tant que marqueur d'identité, ce qui explique pourquoi la variété dominante sous l'angle du statut (la norme hexagonale) n'a pas pu s'imposer dans l'usage québécois (Ryan, 1979 ; Lafontaine, 1997).

\subsection{Compréhensibilité}

Dans le but de savoir si certaines variétés sont jugées plus compréhensibles que d'autres, nous avons demandé à nos juges d'évaluer les différents locuteurs selon la même échelle que celle utilisée pour le test d'attitude proprement dit (de " peu » à " très »). La notion de compréhensibilité telle qu'on l'envisage dans notre étude réfère au niveau de difficulté des juges à comprendre un énoncé produit par un locuteur francophone. Cette définition ne réfère donc qu'à la prononciation, étant donné que les juges devaient lire le même texte. Le tableau 2 montre les cotes moyennes obtenues par groupe de locuteurs et illustre que tous 
sont globalement considérés comme très compréhensibles selon notre grille d'évaluation $(>3,51)$ et qu'encore une fois, la variété québécoise reçoit les cotes les plus positives.

\begin{tabular}{|l|c|c|c|c|c|}
\hline & Québec & France & Algérie & Haïti & Cameroun \\
\hline Compréhensibilité & $\mathbf{5 , 8 1}$ & $\mathbf{5 , 2 7}$ & $\mathbf{5 , 0 6}$ & $\mathbf{4 , 7 5}$ & $\mathbf{4 , 6 7}$ \\
\hline
\end{tabular}

Tableau 2. Moyennes obtenues pour la compréhensibilité des voix francophones.

Afin de savoir si la différence entre les moyennes obtenues était significative, nous avons de nouveau effectué un test $t$. Selon les résultats, les voix québécoises sont considérées significativement plus compréhensibles que les autres groupes (voir tableau 2a en annexe). Les voix françaises sont considérées significativement plus compréhensibles que les voix haïtiennes et camerounaises (voir tableau $2 \mathrm{~b}$ en annexe). En revanche, il n'y a pas de différence significative entre les voix algériennes, haïtiennes et camerounaises (voir tableau $2 \mathrm{c}$ et $2 \mathrm{~d}$ en annexe).

Le classement des variétés testées selon la compréhensibilité indique que les juges perçoivent moins de difficultés de compréhension avec les variétés qui leur sont plus familières, à savoir leur propre variété et celle des Français, omniprésente au Québec depuis longtemps. Cette observation va dans le même sens que celle de Moreau et al. (2007: 37) où les auditeurs québécois jugeaient les voix québécoises scolarisées plus compréhensibles. Ces résultats sont aussi congruents avec les études antérieures indiquant que plus les variétés d'anglais (p. ex. Adank, Evans, Stuart-Smith et Scott, 2009) ou l'accent étranger sont familiers (p. ex. Crowther, Tromimovich, Saito et Isaacs, 2014 ; Munro et Derwing, 1995), plus les jugements de compréhensibilité sont cléments. Van Engen et Peele (2014) affirment que cette tendance peut être due à l'effort cognitif que le locuteur doit déployer pour traiter un contenu linguistique comportant des déviations segmentales ou suprasegmentales par rapport à ce qui est connu et donc présent en mémoire.

Nous avons ensuite vérifié s'il y avait une corrélation entre le niveau de compréhensibilité et les attitudes exprimées par les juges dans le test d'attitudes. Nous avons testé séparément le taux de corrélation entre, d'une part, la compréhensibilité et la solidarité (la corrélation de Spearman égale à $0,377^{* *}$ avec une significativité de $0,000^{* *}$ ) ; d'autre part, la compréhensibilité et le statut (la corrélation de Spearman égale à $0,546^{* *}$ avec une significativité de $0,000^{* * *}$ ). Selon nos résultats, la compréhensibilité était positivement corrélée à l'évaluation des attitudes. Le test de régression a ensuite confirmé que les attitudes des juges étaient d'autant plus positives que les locuteurs étaient jugés compréhensibles, et ce, pour les deux facteurs (solidarité : sig. $0,000^{* * *}, \mathrm{R}^{2}=14 \%$; statut : sig. $0,000^{* * *}, \mathrm{R}^{2}=29 \%$ ). Cette observation va dans le même sens que celle de Kang et Rubin (2009) et de Rubin (1992) qui ont déjà remarqué que les jugements de compréhensibilité peuvent être médiés par des facteurs non linguistiques tels que les attitudes linguistiques entretenues par le juge, les attitudes positives à l'égard de la communauté cible étant corrélées à des jugements de compréhensibilité positifs.

\subsection{Prononciation jugée correcte}

Nous nous sommes ensuite intéressées à l'attitude potentiellement normative des juges à l'égard de la prononciation des différents locuteurs. Nous avons voulu savoir si une norme de prestige endogène émergeait au sein de la communauté québécoise. Autrement dit, estce qu'une hiérarchisation des locuteurs francophones est formulée par les juges en fonction de la correction de leur prononciation ? Pour cela, nous avons demandé à nos juges d'évaluer la prononciation des locuteurs sur une échelle de 1 à 6 (de "peu » à 《très » 
correcte). Le tableau 3 présente les cotes moyennes obtenues par groupe de locuteurs et indique que tous sont globalement considérés comme ayant une prononciation très correcte selon notre grille d'évaluation $(>3,51)$.

\begin{tabular}{|l|c|c|c|c|c|}
\hline & Québec & France & Algérie & Haïti & Cameroun \\
\hline Prononciation « correcte » & $\mathbf{5 , 6 7}$ & $\mathbf{5 , 3 1}$ & $\mathbf{5 , 1 0}$ & $\mathbf{4 , 8 0}$ & $\mathbf{4 , 5 8}$ \\
\hline
\end{tabular}

Tableau 3. Moyennes obtenues pour les prononciations considérées comme correctes.

Les résultats $\mathrm{du}$ test $\mathrm{t}$ (voir tableau 3 en annexe) permettent d'observer que la prononciation des locuteurs québécois est encore jugée significativement plus correcte que celle de tous les autres groupes. La prononciation des Français est jugée significativement plus correcte que celle des Haïtiens et des Camerounais. En revanche, il n'y a pas de différence significative entre les voix algériennes, haïtiennes et camerounaises (voir tableau $3 \mathrm{c}$ et $3 \mathrm{~d}$ en annexe). La préférence des voix québécoises par rapport aux autres, selon le critère de la correction, confirme les observations de Pustka et al. (2019:40) qui ont identifié eux aussi l'accent de la ville de Québec comme étant celui jugé le plus correct par rapport à six autres accents francophones (parisien, suisse, belge, africain, acadien, montréalais). Un résultat semblable se dégage de l'étude de Moreau et al. (2007 : 32) qui révèle également la tendance à mieux évaluer le français parlé des Québécois scolarisés par rapport aux variétés de français parlées des Français, Sénégalais, Suisses, Tunisiens et Belges.

Nous avons ensuite vérifié s'il y avait une corrélation entre le niveau de correction de la prononciation et les attitudes exprimées par les juges dans le test d'attitudes. Nous avons testé séparément le taux de corrélation entre, d'une part, la correction de la prononciation et la solidarité (la corrélation de Spearman égale à $0,291^{* *}$ avec une significativité de $0,000^{* *}$ ) et, d'autre part, la correction de la prononciation et le statut (la corrélation de Spearman égale à $0,464 * *$ avec une significativité de $0,000^{* * *}$ ). Selon nos résultats, la correction de la prononciation était positivement corrélée à l'évaluation des attitudes. Le test de régression a ensuite confirmé que les attitudes des juges étaient d'autant plus positives que la prononciation des locuteurs était jugée correcte. Néanmoins, l'impact de la correction de la prononciation semble être plus important pour le facteur statut $\left(\mathrm{R}^{2}=22 \%\right.$, sig. $\left.0,000^{* * *}\right)$ que pour le facteur solidarité $\left(\mathrm{R}^{2}=8 \%\right.$, sig. $\left.0,000^{* * *}\right)$. En résumé, la mesure de la correction nous permet de confirmer une attitude incontestablement positive à l'égard des voix québécoises, ce qui suggère l'existence d'une norme endogène.

\subsection{Variation situationnelle}

Les locuteurs vivent des situations de communication différentes au quotidien. Ils interagissent avec des membres de leurs familles, rencontrent des amis, discutent avec des collègues (Émond, 2018 : 85). Nous nous sommes demandé quelle variété géographique serait la mieux évaluée par les juges québécois dans trois contextes différents selon le niveau de formalité, à savoir la sphère privée, la sphère professionnelle et la sphère publique. Pour ce faire, nous avons calculé le pourcentage de juges qui seraient d'accord pour choisir telle ou telle personne dans le contexte donné.

\subsubsection{Sphère privée : Intégration dans la vie sociale québécoise}

En vue de la question de l'intégration des nouveaux arrivants dans la vie sociale québécoise, nous avons demandé à nos juges de décider si les différents locuteurs entendus pourraient devenir leurs amis. L'objectif était de savoir quelle variété serait la plus 
valorisée dans un contexte informel. Le tableau 4 ci-dessous affiche le pourcentage de juges ayant donné une réponse affirmative.

\begin{tabular}{|l|c|c|c|c|c|}
\hline & Québec & Haïti & Algérie & Cameroun & France \\
\hline Relations amicales & $\mathbf{9 4 \%}$ & $\mathbf{8 1 \%}$ & $\mathbf{6 9} \%$ & $\mathbf{6 7 \%}$ & $\mathbf{6 5 \%}$ \\
\hline
\end{tabular}

Tableau 4. Volonté d'établir des relations amicales avec des locuteurs francophones.

Les pourcentages affichés dans le tableau 4 montrent que les juges expriment une très nette préférence pour les locuteurs du FQ dans le contexte amical. Au vu de ces résultats, nous avons voulu mieux comprendre à quoi était liée une telle différence entre les différents pourcentages. C'est la raison pour laquelle nous nous sommes demandé si les cotes attribuées aux locuteurs en fonction du facteur solidarité dans le cadre du test d'attitudes (voir tableau 1) reflétaient les résultats obtenus pour le contexte des relations amicales à la suite du dépouillement du questionnaire. La figure 2 met les données tirées du tableau 1 et 4 en relation.

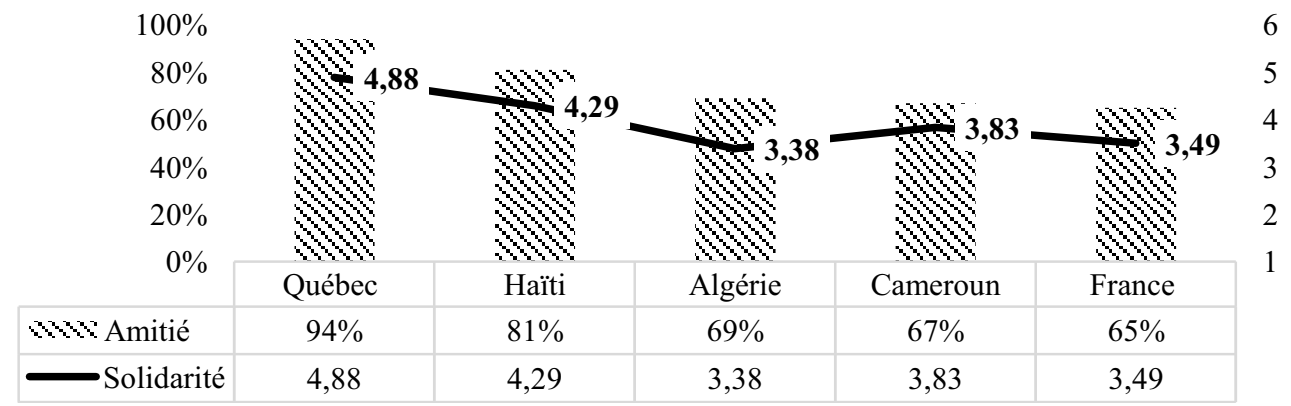

Fig. 1. Lien entre le facteur solidarité et la volonté d'établir des relations amicales.

La figure 2 montre que les raisons socioaffectives (facteur solidarité lié à la personnalité des locuteurs) jouent potentiellement un rôle primordial dans les évaluations des différents locuteurs en tant qu'amis potentiels. En effet, le pourcentage correspondant à la volonté d'établir des relations amicales avec les différents locuteurs francophones est le même que celui de la moyenne des cotes attribuées aux locuteurs en fonction du facteur solidarité. Autrement dit, plus les locuteurs sont considérés sympathiques, dynamiques et sociables, plus ils seraient facilement intégrés dans la sphère sociale. Le test de régression linéaire montre que notre modèle est adéquat, puisque le coefficient de détermination $\mathrm{R}^{2}=90 \%$, sig. $0,01^{*}$.

\subsubsection{Sphère professionnelle : Insertion dans le milieu de travail}

Selon le portrait statistique des immigrants, ces derniers sont plus nombreux à chercher du travail et à intégrer la vie professionnelle que les citoyens eux-mêmes nés au pays (Bourque, 2018). À la lumière de ces observations, nous avons demandé aux juges d'indiquer s'ils pouvaient s'imaginer travailler avec les personnes entendues. En effet, il a été démontré que le capital linguistique détermine l'accès aux ressources sociales et publiques (Harrison, 2014). Dans un grand nombre de domaines, le travail est souvent fondé sur la communication et aussi, par conséquent, sur la langue (Harrison, 2014 : 256). Dans la sphère professionnelle, à commencer par les entretiens d'embauche, ce sont souvent les capacités linguistiques du demandeur d'emploi et la perception de son appartenance ethnique par l'employeur qui déterminent le succès de son insertion dans le 
milieu de travail, plus que ses compétences professionnelles. Comme le souligne également Bourdieu (1977 : 563) : « vocal features such as accent and speech style serve as signifiers of status and credibility ». La crédibilité semble être conditionnée non seulement par l'acceptabilité de tel ou tel accent, mais aussi par l'autorité de la personne qui parle. L'appartenance à telle ou telle position sociale serait donc davantage conditionnée par la forme de l'énoncé du locuteur que par le fond (Harrison, 2014: 263). Le tableau 5 cidessous affiche le pourcentage de juges ayant donné une réponse affirmative.

\begin{tabular}{|l|c|c|c|c|c|}
\hline & Québec & France & Haïti & Algérie & Cameroun \\
\hline Relations professionnelles & $\mathbf{9 8 \%}$ & $\mathbf{9 6} \%$ & $\mathbf{8 8} \%$ & $\mathbf{8 5 \%}$ & $\mathbf{8 3} \%$ \\
\hline
\end{tabular}

Tableau 5. Volonté d'établir des relations professionnelles avec des locuteurs francophones.

Les pourcentages affichés dans le tableau 5 montrent que les juges expriment encore une fois une préférence pour les locuteurs du FQ. De manière globale, nous pouvons observer que les pourcentages sont considérablement plus élevés que dans le tableau précédent portant sur l'établissement des relations amicales. Il s'agit d'une observation significative. En effet, selon le test de régression, les moyennes sont significativement plus élevées pour les relations amicales que pour les relations professionnelles (le coefficient de détermination $\mathrm{R}^{2}=90 \%$, sig. $\left.0,006^{*}\right)$. Le principal changement est visible pour les locuteurs du français de France $(96 \%$ ) qui dépassent cette fois-ci les trois autres groupes francophones, arrivant presque aux mêmes résultats que les locuteurs du FQ (98\%). Nous pouvons en conclure que les juges québécois hésiteraient moins à accepter la personne en tant que collègue qu'à lier une amitié avec elle.

Ensuite, nous avons voulu vérifier si les cotes attribuées aux locuteurs en fonction du facteur statut reflétaient les résultats obtenus pour le contexte des relations professionnelles. La figure 3 met les données tirées du tableau 1 et 5 en relation.

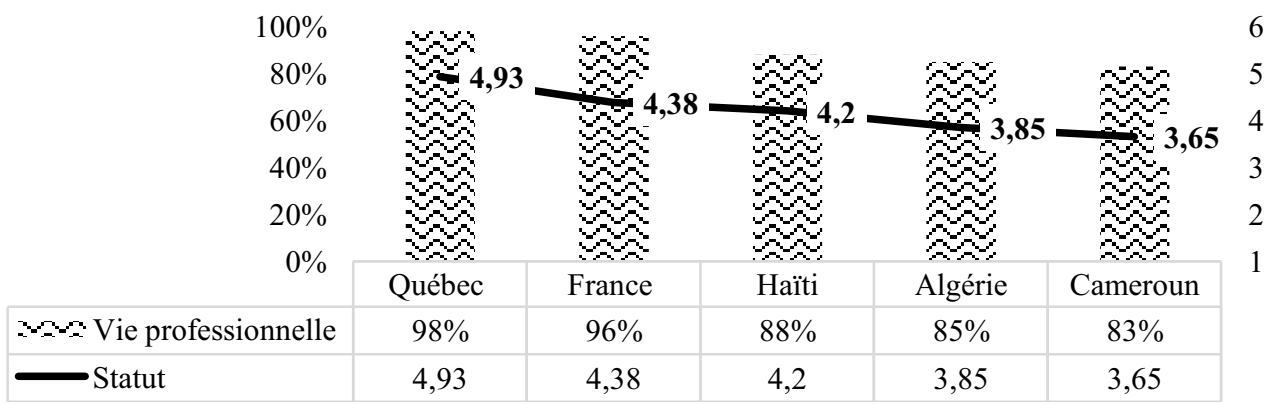

Fig. 3. Lien entre le facteur statut et la volonté d'établir des relations professionnelles.

La figure 3 montre que ce sont encore une fois les locuteurs québécois qui sont préférés par les juges. Cependant, les écarts entre les pourcentages sont moindres que dans la figure précédente. En effet, le pourcentage correspondant à la volonté d'établir des relations professionnelles avec les immigrants est proportionnel aux cotes attribuées aux locuteurs en fonction du facteur statut. Les juges québécois ont donc des appréciations très favorables à l'égard de leur propre communauté linguistique (98\% et cote de 4,93), mais aussi à l'égard des autres groupes francophones. Selon notre grille d'évaluation, nous pouvons considérer que les appréciations globales envers les autres francophones sont plutôt positives. Cela laisse croire que les Québécois accepteraient les locuteurs francophones dans la vie professionnelle, probablement avec moins de prudence que dans la vie privée. Le test de régression linéaire montre que notre résultat est adéquat, puisque le coefficient de détermination $\mathrm{R}^{2}=95 \%$, sig. $0,016^{*}$. En effet, comme nous l'avons démontré ci-dessus, 
les juges attribuent les cotes plus élevées au facteur statut et sont plus exigeants quant à la solidarité (voir 3.1).

En résumé, les questions quant aux relations amicales et professionnelles dévoilent un lien fort, d'une part, entre les jugements concernant la personnalité des voix et leur intégration dans la vie sociale et, d'autre part, entre les jugements concernant les compétences attribuées aux voix et leur intégration dans la vie professionnelle.

\subsubsection{Sphère publique : Enseignement du français langue première}

Les situations les plus formelles, qu'on rencontre surtout dans la sphère publique, sont caractérisées par l'application d'une norme prescriptive, correspondant à la variété légitime, considérée comme standard et véhiculée par des instances normatives telles que l'école. Cette dernière transmet souvent une image de la langue comme étant unifiée, ce qui entre en concurrence avec la diversité des usages appris dans le milieu ambiant, dont certains finissent par être stigmatisés (Remysen, 2018). Au vu des questionnements sur la langue enseignée dans les écoles au Québec, nous avons demandé aux juges si les personnes entendues pouvaient travailler comme enseignants de français à l'école secondaire. Le tableau 6 montre le pourcentage de juges ayant donné une réponse affirmative.

\begin{tabular}{|l|c|c|c|c|c|}
\hline & Québec & France & Algérie & Haïti & Cameroun \\
\hline $\begin{array}{l}\text { Enseignement du } \\
\text { français }\end{array}$ & $\mathbf{8 5} \%$ & $\mathbf{7 5 \%}$ & $\mathbf{6 9 \%}$ & $\mathbf{6 0} \%$ & $\mathbf{4 8 \%}$ \\
\hline
\end{tabular}

Tableau 6. Enseignement du français à l'école secondaire.

Le tableau 6 montre que $85 \%$ des évaluateurs seraient d'accord pour que les locuteurs québécois occupent un poste d'enseignant de français à l'école secondaire. Il s'agit d'un résultat intéressant qui indique que la norme à privilégier dans l'enseignement se situe, pour la majorité des juges, à l'intérieur du Québec, du moins pour la langue première. Ce constat a déjà été formulé par Moreau et al. (2007 : 24) où les auditeurs québécois situaient les Québécois fortement scolarisés au premier rang. Le résultat pourrait être différent si nous avions inclus l'enseignement $\mathrm{du}$ français langue étrangère ou seconde dans notre questionnaire. Ainsi, une étude récente révèle que les particularités du FQ n'y sont pas encore totalement acceptées (Duchemin, 2017). Il reste que le taux d'accord obtenu pour cette question est considérablement plus bas que les taux obtenus pour la sphère privée (3.4.1) et la sphère professionnelle (3.4.2). En conséquence, les juges se montrent plus sévères envers le contexte d'enseignement qu'envers les deux autres contextes testés. Les résultats du test de régression - ayant pour but de vérifier si les cotes attribuées aux locuteurs en fonction des facteurs solidarité et statut ont un impact sur les résultats obtenus pour le contexte de l'enseignement - le confirment : il n'y a pas de lien entre les résultats du tableau 6 et les données tirées du tableau 1, puisque le coefficient de détermination $\mathrm{R}^{2}=$ $74 \%$, sig. 0,06 pour le facteur solidarité et $\mathrm{R}^{2}=31 \%$, sig. 0,33 pour le facteur statut. La question concernant l'enseignement ne révèle donc pas de lien entre les jugements sur la personnalité des voix et la capacité perçue en tant qu'enseignant.

\section{Conclusion}

Nos résultats révèlent que les voix québécoises se démarquent considérablement des autres en recevant des évaluations plus positives, tant dans le test d'attitudes que dans le questionnaire. Cette observation va dans le même sens que celles des études récentes qui ont déjà constaté une amélioration des attitudes à l'égard du FQ au cours des dernières décennies (Evans, 2002 ; Remysen, 2004 ; Moreau et al., 2007; Chalier, 2014 et 2018; 
Pustka et al., 2019). Nos résultats indiquent en effet que la norme endogène québécoise est en train de se stabiliser et qu'elle jouirait désormais non seulement d'un prestige latent, mais aussi d'un prestige manifeste, plus grand même que celui de la variété française, ce qui correspond aux constats de Chalier (2014 et 2018) et de Pustka et al. (2019).

Il est intéressant de noter que les résultats du test sont en lien étroit avec ceux du questionnaire à réponse unique, ce qui suggère que les attitudes des juges sont aujourd'hui plus univoques qu'elles ne l'étaient dans le passé (Tremblay, 1990 ; Remysen, 2004). L'ancien prestige du français hexagonal a cependant laissé des traces puisqu'il a été mieux évalué que les trois autres variétés non québécoises quant au statut, qui est d'ailleurs le seul facteur où la différence entre la variété québécoise et la variété française n'est pas toujours significative. Cette représentation est sans doute héritée d'une époque où l'on opposait le français hexagonal, considéré comme langue de prestige, et le FQ, langue sans statut de qualité (Ostiguy et Reinke, 2015).

Quant aux attitudes à l'égard des cinq variétés de français parlées dans la ville de Québec, elles sont aussi globalement positives. Étant donné que la ville de Québec a depuis longtemps la réputation d'être relativement conservatrice et que la diversité ethnolinguistique y est un phénomène récent, il s'agit là d'une observation remarquable et encourageante eu égard à l'accueil des immigrants qui posséderaient le capital linguistique requis pour leur intégration socioprofessionnelle. En revanche, les différences entre les résultats du questionnaire concernant les sphères amicale, professionnelle et publique permettent de croire que l'intégration dans la vie professionnelle serait plus facile pour les immigrants que l'intégration dans la vie sociale ; nos résultats indiquant une moins grande ouverture. En effet, nos résultats concernant la configuration des rapports entre la société d'accueil et la personne immigrante vont dans le même sens que ceux observés dans d'autres disciplines telles que la sociologie et la psychologie sociale. Ainsi, comme le définit la sociologie, la sphère publique est régie par des normes et des valeurs partagées tandis que la sphère privée est le domaine réservé à l'identité personnelle (Chandler et Munday, 2011). En conséquence, accueillir la personne immigrante dans les domaines périphériques ou publics (p.ex. le travail, l'école) s'avère être plus facile que dans les domaines privés où s'incarnent davantage de traditions et de valeurs (p.ex. la famille, l'amitié) (Velandia-Coustol et Rojas-Tejada, 2018). Autrement dit, la sphère privée semble donc être moins perméable à "l'étrangeté » que la sphère publique. À la lumière de ces constats on comprend mieux que l'ouverture au multiculturalisme du Québec et du Canada ne semble pas franchir le seuil du chez-soi.

Rappelons que nos résultats ne valent que pour le groupe de jeunes représenté par notre échantillonnage et devraient être validés par une étude de plus grande envergure. Par contre, cette apparente limite de l'étude constitue aussi une force, puisque le comportement des jeunes adultes est un bon indicateur de l'évolution des attitudes dans la société.

\section{Références bibliographiques}

Adank, P., Evans, B. G., Stuart-Smith, J. et Scott, S. K. (2009). Comprehension of familiar and unfamiliar native accents under adverse listening conditions. Journal of Experimental Psychology: Human Perception and Performance, 35, 520-529.

Avanzi, M. (2016). Rôle de la prosodie dans la perception de variétés régionale et nonnative du français. Langages, 202 (2), 35-46.

Avanzi, M. et Boula de Mareüil, P. (2017). Identification of regional French accents in (northern) France, Belgium and Switzerland. Journal of Linguistic Geography, 1 (5), $17-40$. 
Avanzi, M. et Boula de Mareüil, P. (2019). Peut-on identifier perceptivement huit accents régionaux en français? La réponse des sciences participatives. Glottopol, 31, 53-73.

Bouchard, P. et Maurais, J. (1999). La norme et l'école. L'opinion des Québécois. Dans O. Conrad (dir.), La norme du français au Québec. Perspectives pédagogiques, Terminogramme, 91-92, 91-116.

Bourque, F. (2018). Portrait statistique des immigrants au Québec. Récupéré le 6 décembre 2019 du site du journal Le Soleil: https://www.lesoleil.com/chroniques/portraitstatistique-des-immigrants-de-quebec-1d03725cbb3a226815e18e7e65fd8a $9 \mathrm{~b}$.

Bourdieu, P. (1977). The economics of linguistic exchanges. Social Science Information, 16 (6), 645-668.

Calvet, L.-J. (2017). La sociolinguistique. Paris: Presses Universitaires de France/Humensis.

Chalier, M. (2014). Quel standard québécois? Une étude perceptive (mémoire de maîtrise, Ludwig-Maxmilians-Universität München, Allemagne).

Chandler, D., et Munday, R. (2011). Public and private spheres. Dans A Dictionary of Media and Communication. Oxford University Press. Récupéré le 29 mars 2020 du : https://www.oxfordreference.com/view/10.1093/acref/9780199568758.001.0001/acre f-9780199568758-e-2195.

Chalier, M. (2018). Quelle norme de prononciation au Québec ? Attitudes, représentations et perceptions. Dans Langage et société, 1 (163), 121-144.

Crowther, D., Trofimovich, P., Saito, K. et Isaacs, T. (2014). Second language comprehensibility revisited: Investigating the effects of learner background. TESOL Quarterly, 49 (4), 814-37.

D'Anglejan, A., Tucker, G. R. et Centre for Franco-Ontarian Studies (1972). Sociolinguistic correlates of speech style in Quebec. Toronto : Centre de recherches en éducation franco-ontarienne, Institut d'études pédagogiques de l'Ontario.

Dragojevic, M., Giles, H. et Watson, B. M. (2013). Language ideologies and language attitudes: a foundational framework. Dans H. Giles et B. M. Watson (dir.), The Social Meanings of Language, Dialect and Accent: International Perspectives on Speech Styles (p. 1-25). New York : Peter Lang.

Duchemin, M. (2017). Les représentations associées aux français nationaux, aux espaces géographiques et aux locuteurs dans les manuels de français langue étrangère et de français langue seconde: étude comparée entre la France et le Québec. Revue canadienne de linguistique appliquée, 20 (2), 51-70.

Émond, C. (2018). Français québécois parlé : pour un enseignement basé sur une approche linguistique de la situation de communication. Dans N. Vincent et S. Piron (dir.), $L a$ linguistique et le dictionnaire au service de l'enseignement du français au Québec. Mélanges offerts à Hélène Cajolet-Laganière (p. 81-109). Montréal : Nota Bene.

Evans, B. (2002). Attitudes of Montreal Students Towards Varieties of French. Dans Long, D. et Preston, D. (dir.). Handbook of Perceptual Dialectogy (vol. 2, p. 71-93). Amsterdam/Philadelphie: John Benjamins.

Giles, H. et Billings, A. (2004). Assessing language attitudes: Speaker evaluation studies. Dans A. Davies et C. Elder (dir.), Handbook of Applied Linguistics (p. 187-209). Oxford: Basil Blackwell.

Genesee, F. et Holobow, N. (1989). Change and Stability in Intergroup Perceptions. Journal of Language and Social Psychology, 8 (1), 17-38. 
Goldman, J.-P., Avanzi, M. et Schwab, S. (2014). Perception de l'accent régional dans cinq variétés de français parlées en Suisse romande. Nouveaux cahiers de linguistique française, 31, 285-296.

Fuertes, J. N., Gottdiener, W. H., Martin, H., Gilbert, T. C. et Giles, H. (2012). A metaanalysis of the effects of speakers' accents on interpersonal evaluations. European Journal of Social Psychology, 42 (1), 120-133.

Harrison, G. (2014). Accent and 'Othering' in the Workplace. Dans J. Levis (dir.), Social Dynamics in Second Language Accent (p. 255-272). Berlin : De Gruyter.

Immigration, Réfugiés et Citoyenneté Canada (2019). Atteindre nos objectifs : Stratégie en matière d'immigration francophone. Récupéré le 5 avril 2019 du : https://www.canada.ca/content/dam/ircc/documents/pdf/francais/organisation/publicat ions-guides/strategie-immigration-francophone/franco-immigr-strateg-fra.pdf.

Kang, O. et Rubin, D. L. (2009). Reverse linguistic stereotyping: Measuring the effect of listener expectations on speech evaluation. Journal of Language and Social Psychology, 28, 441-456.

Kang, O. et Rubin, D. (2014). Listener Expectations, Reverse Linguistic Stereotyping, and Individual Background Factors in Social Judgments and Oral Performance Assessment. Dans J. Levis (dir.), Social Dynamics in Second Language Accent (p. 239-254). Berlin : De Gruyter.

Kircher, R. (2014). Thirty Years after Bill 101: A Contemporary Perspective on Attitudes Towards English and French in Montreal. Revue canadienne de linguistique appliquée, 17 (1), 20-50.

Laberge, S. et Chiasson-Lavoie, M. (1971). Attitudes face au français parlé à Montréal et degrés de conscience de variables linguistiques. Dans R. Darnell (dir.), Linguistic diversity in Canadian society (p. 89-126). Edmonton : Linguistic Research Inc.

Labov, W. (1976). Sociolinguistique. Paris : Éditions de Minuit.

Lafontaine, D. (1997). Attitudes linguistiques. Dans Moreau, M.-L. (dir.), Sociolinguistique. Les concepts de base (p. 56-60). Sprimont : Mardaga.

Lambert, W. E., Hodgson, R. C., Gardner, R. C. et Fillenbaum, S. (1960). Evaluational reactions to spoken languages. The Journal of Abnormal and Social Psychology, $60(1), 44-51$.

Lappin, K. (1982). Évaluation de la prononciation du français montréalais : étude sociolinguistique. Revue québécoise de linguistique, 11 (2), 93-112.

Laur, E. (2001). Perceptions linguistiques à Montréal (thèse de doctorat, Université de Montréal, Québec, Canada).

Laur, E. (2008). Contribution à l'étude des perceptions linguistiques. La méthodologie des faux-couples revisitée. Montréal : Office québécois de la langue française.

Lazaridis, A., Goldman, J.-P., Avanzi, M. et Garner, P. (2014). Syllable-based Regional Swiss French Accent Identification using Prosodic Features. Nouveaux cahiers de linguistique française, 31, 297-307.

Lindemann, S. (2003). Koreans, Chinese or Indians? Attitudes and ideologies about nonnative English speakers in the United States. Journal of Sociolinguistics, 7 (3), 348364.

Lindemann, S., Litzenberg, J. et Subtirelu, N. (2014). Problematizing the Dependence on L1 Norms in Pronunciation Teaching: Attitudes toward Second-language Accents. Dans J, Levis et A. Moyer (dir.), Social Dynamics in Second Language Accent (171194). Berlin : De Gruyter. 
Maurais, J. (2008). Les Québécois et la norme : l'évaluation par les Québécois de leurs usages linguistiques. Montréal : Office québécois de la langue française.

Méar-Crine, A. et Leclerc, T. (1976). Attitudes des adolescents canadiens-français vis-à-vis du franco-québécois et du français académique. Cahiers de linguistique, 6, 155-170.

McKenzie, R. M. (2010). Social Psychology of English as a Global Language. Dordrecht/New York : Springer.

Moreau, M.-L., Bouchard, P., Gadet, F., Demartin, S., Guerin, E., Harmegnies, B., ...Tyne, H. (2007). Les accents dans la francophonie. Une enquête internationale. Français et Société, 16.

Munro, M. J. et Derwing, T. M. (1995). Foreign accent, comprehensibility, and intelligibility in the speech of second language learners. Language Learning, 45, 7397.

Nejjari, W., Gerritsen, M., van Hout, R. et Planken, B. (2019). Refinement of the matchedguise technique for the study of the effect of non-native accents compared to native accents. Lingua, 219, 90-105.

Noël, D. (1980). Le français parlé : analyse des attitudes des adolescents de la ville de Québec selon les classes sociales. Québec: Centre international de recherche sur le bilinguisme.

Ochs, E. et Schieffelin, B. (2017). Language socialization: An historical overview. Dans P. Duff et S. May (dir.), Language Socialization (p. 3-16). New York: Springer.

Ostiguy, L. et Reinke, K. (2015). La langue du doublage québécois : un français « idéal» fabriqué au Québec. Dans K. Sarkowsky, R.-O. Schultze et S. Schwarze (dir.). Migration - Regionalisation - Citizenship: Comparing Canada and Europe (p. 231252). Wiesbaden: Springer VS.

Paveau, M.-A. (2008). Le parler des classes dominantes, objet linguistiquement incorrect ? Dialectologie perceptive et linguistique populaire. Éla. Études de linguistique appliquée, 150, 137-156.

Perrien J., Cheron E. J. et Zins M. (1984). Recherche en marketing : méthodes et décisions. Montréal : Gaëtan Morin.

Pöll, B. (2005). Le français langue pluricentrique? Études sur la variation diatopique d'une langue standard. Francfort-sur-le-Main : Peter Lang.

Pöll, B. (2017). Normes endogènes, variétés de prestige et pluralité normative. Dans U. Reutner (dir.), Manuel des francophonies. Berlin/Boston : De Gruyter.

Porter, I. (2017). Le Québec peine à attirer des immigrants francophones. Récupéré le 25 octobre $2018 \mathrm{du}$ site $\mathrm{du}$ journal Le Devoir: https : //www.ledevoir.com/politique/quebec/511384/le-quebec-peine-a-attirer-desimmigrants-francophones.

Preston, M. S. (1963). Evaluational reactions to English, Canadian French, and European French voices (mémoire de maîtrise non publié, Université McGill, Québec, Canada).

Pustka, E., Bellonie, J.-D., Chalier, M. et Jansen, L. (2019). 'C'est toujours l'autre qui a un accent': le prestige méconnu des accents du Sud, des Antilles et du Québec. Glottopol, 31, 27-52.

Reinke, K. (2000). La norme phonétique du français québécois : les attitudes des Québécois par rapport à leur français. Dans Actes des XIII Journées de linguistique (p. 185-195). Québec: Faculté des lettres et des sciences humaines, Université Laval et Centre universitaire de recherche en aménagement linguistique. 
Remysen, W. (2004). La variation linguistique et l'insécurité linguistique : le cas du français québécois. Dans P. Bouchard, (dir.). La variation dans la langue standard. Actes du colloque tenu les 13 et 14 mai 2002 à l'Université Laval dans le cadre du $70^{e}$ Congrès de l'ACFAS (p. 23-36). Québec : Office québécois de la langue française.

Remysen, W. (2018). L'insécurité linguistique à l'école : un sujet d'étude et un champ d'intervention pour les sociolinguistes. Dans N. Vincent et S. Piron (dir.), La linguistique et le dictionnaire au service de l'enseignement du français au Québec (p. 25-59). Montréal : Nota Bene.

Remysen, W. (2014). Les Québécois perçoivent-ils le français montréalais comme une variété topolectale distincte ? Résultats d'une analyse perceptuelle exploratoire. Revue canadienne de linguistique, 59 (1),109-135.

Remysen, W. et Reinke, K. (2014). Introduction : regards croisés sur les communautés linguistiques de Montréal. Revue canadienne de linguistique, 59 (1) iii-vi.

Rousseau, M. (2012). Changement de langue, changement de voix ? Une étude comparative de l'effet du statut des langues anglaise et française au Québec sur la prosodie de la voix de locuteurs bilingues (mémoire de maîtrise, Université du Québec à Montréal, Canada).

Rubin, D. L. (1992). Nonlanguage factors affecting undergraduates' judgments of nonnative English-speaking teaching assistants. Research in Higher Education, 33, 511531.

Ryan, E.-B. (1979). Why do low prestige varieties persist? Dans H. Giles et R. St. Clair (dir.), Language and Social Psychology (p. 145-157). Oxford : Basil Blackwell.

Statistique Canada (2016). Immigration et diversité culturelle du Canada. Récupéré le 18 mars 2019 du site de Statistique Canada: https://www12.statcan.gc.ca/nhsenm/2011/as-sa/99-010-x/99-010-x2011001-fra.cfm.

Tremblay, L. (1990). Attitudes linguistiques et perception sociale de variables phonétiques. Revue québécoise de linguistique théorique et appliquée, 9 (3), 197-221.

Yook, C. et Lindemann, S. (2013). The role of speaker identification in Korean university students' attitudes towards five varieties of English. Journal of Multilingual and Multicultural Development, 34 (3), 279-296.

Van Engen, K. J. et Peelle, J. E. (2014). Listening effort and accented speech. Frontiers in Human Neuroscience, 8 (577).

Velandia-Coustol, C.-R., Navas-Luque, M. \& Rojas-Tejada, A.-J. (2018). Le Modèle Amplifié d'Acculturation Relative (MAAR). État des lieux et perspectives de recherche. Revue européenne des migrations internationales, 34(2-3), 299-317. 

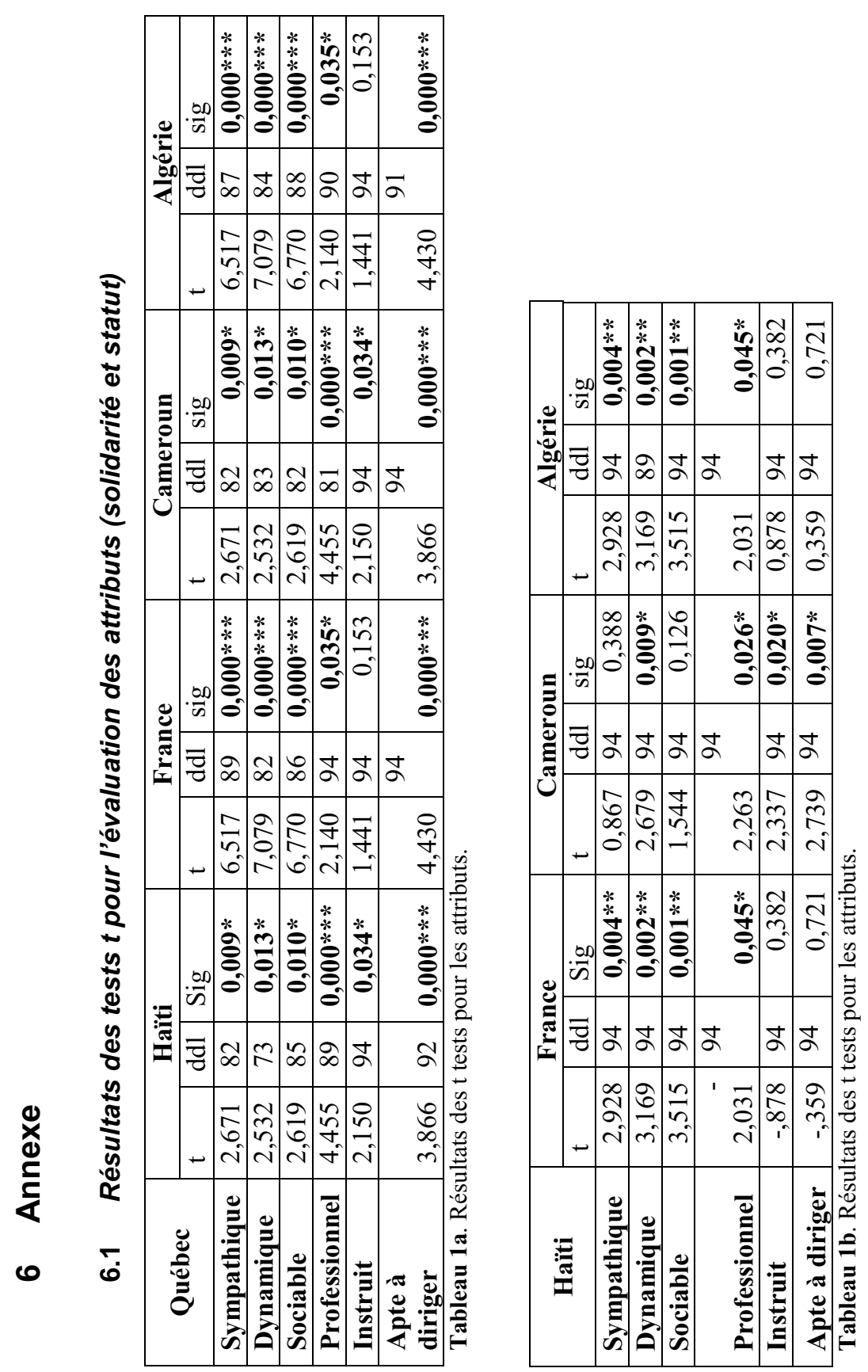

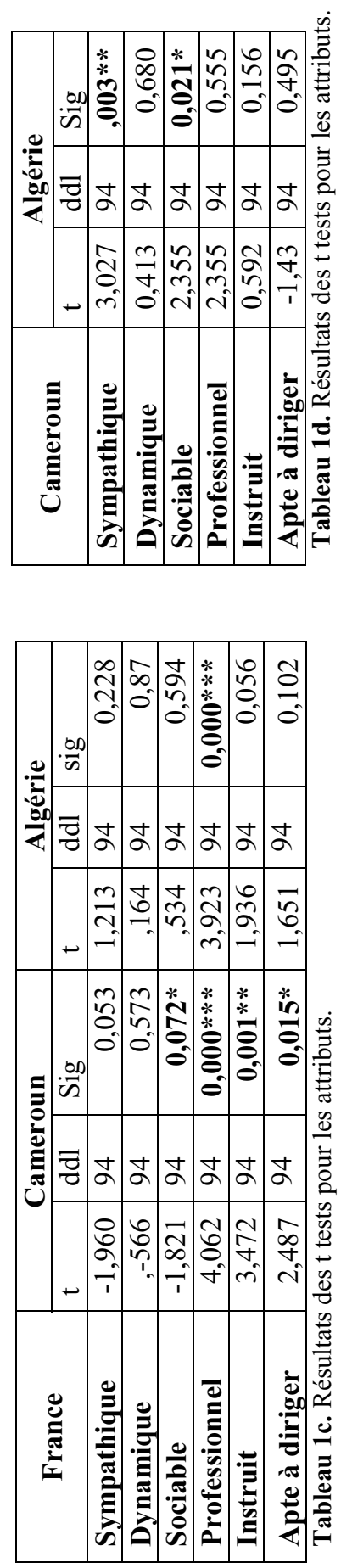
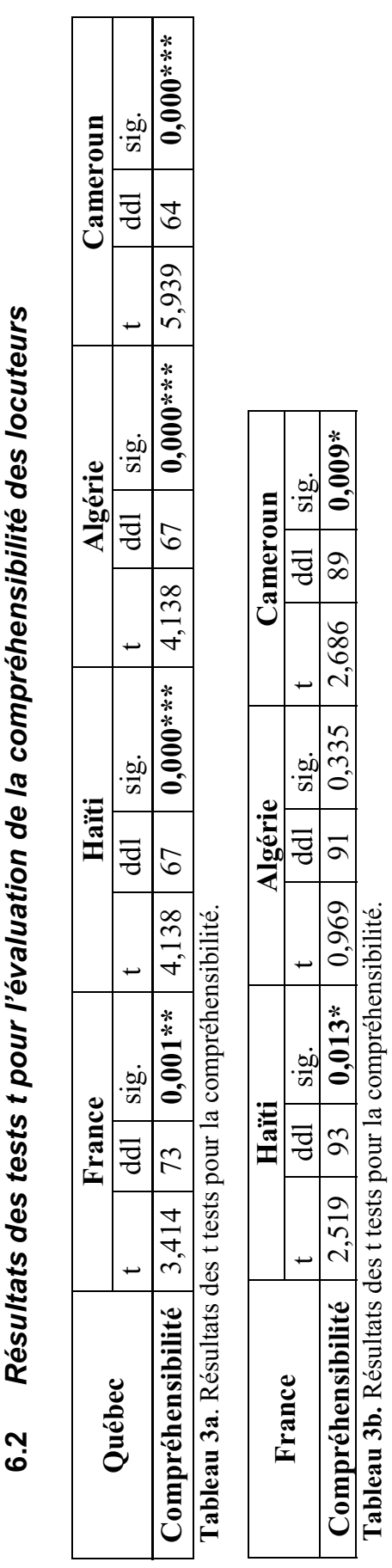

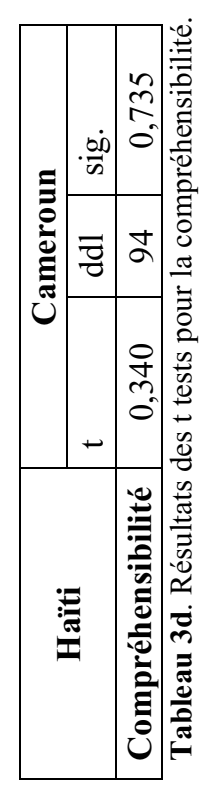




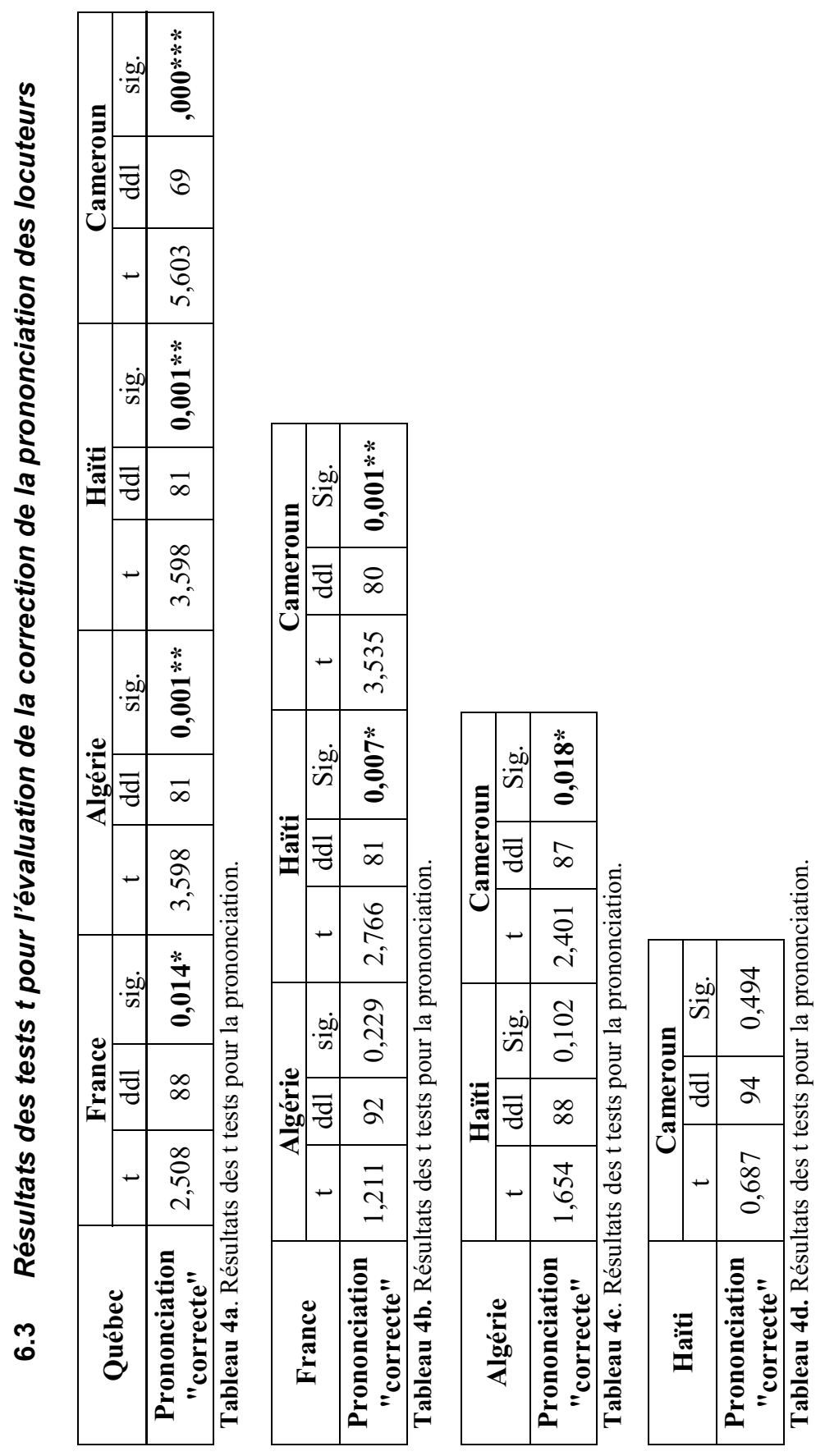




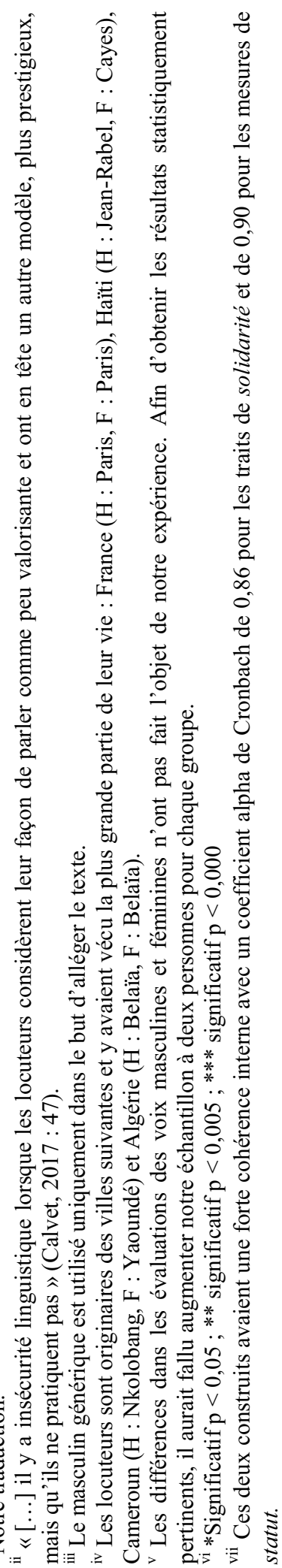

